\title{
ATTRACTORS FOR STOCHASTIC LATTICE DYNAMICAL SYSTEMS WITH A MULTIPLICATIVE NOISE
}

\author{
TOMÁS CARABALLO AND KENING LU
}

\begin{abstract}
In this paper, we consider a stochastic lattice differential equation with diffusive nearest neighbor interaction, a dissipative nonlinear reaction term, and a multiplicative white noise at each node. We prove the existence of a compact global random attractor which pulled back attracts tempered random bounded sets.
\end{abstract}

\section{Introduction}

This paper is devoted to the long term behavior of the following stochastic lattice differential equation

$$
\frac{d u_{i}(t)}{d t}=\nu\left(u_{i-1}-2 u_{i}+u_{i+1}\right)-\lambda u_{i}-f_{i}\left(u_{i}\right)+g_{i}+\sum_{j=1}^{N} c_{j} u_{i} \circ \frac{d w_{j}(t)}{d t}, \quad i \in \mathbb{Z},
$$

where $u=\left(u_{i}\right)_{i \in \mathbb{Z}} \in \ell^{2}, \mathbb{Z}$ denotes the integer set, $\nu$ and $\lambda$ are positive constants, $f_{i}$ is a smooth function satisfying a dissipative condition, $g=\left(g_{i}\right)_{i \in \mathbb{Z}} \in \ell^{2}, c_{j} \in \mathbb{R}$, for $j=1, \ldots, N$, and $w_{j}$ are mutually independent Brownian motions, where o denotes the Stratonovich sense in the stochastic term.

Stochastic lattice differential equations arise naturally in a wide variety of applications where the spatial structure has a discrete character and uncertainties or random influences, called noises, are taken into account. These systems are used to model such systems as cellular neural networks with applications to image processing, pattern recognition, and brain science $[15,16,17,18]$. They are also used to model the propagation of pulses in myelinated axons where the membrane is excitable only at spatially discrete sites. In this case, $u_{i}$ represents the potential at the $i$-th active site; see for example, [7], [8], [39], [36], [30, 31]. Lattice differential equations can also be found in chemical reaction theory $[24,28,33]$. Equation (1.1) is a one-dimensional lattice system with diffusive nearest neighbor interaction, a dissipative nonlinear reaction term and a multiplicative white noise at each node. This may be the result of an environmental effect on the whole domain of the system. The system with an additive noise was studied in Bates et al. [5].

Recently, there are many works on deterministic lattice dynamical systems. For traveling waves, we refer the readers to $[11,34,12,42,1,4]$ and the references therein. The chaotic properties of solutions for such systems have been investigated by [11] and [14, 40, 13, 22]. In the absence of the white noise, the existence of a global attractor for lattice differential equation (1.1) was established in $[6]$.

1991 Mathematics Subject Classification. Primary: 60H15; Secondary: 34C35, 58F11, 58F15, 58F36.

Key words and phrases. stochastic lattice differential equations, random attractors, multiplicative noise. This work was partially supported by Ministerio de Educación y Ciencia (Spain) MTM2005-01412 (T. Caraballo) and NSF0200961, NSF0401708, and NSFC10371083 (K. Lu). 
The study of global random attractors was initiated by Ruelle [37]. The fundamental theory of global random attractors for stochastic partial differential equations was developed by Crauel, Debussche, and Flandoli [20], Crauel and Flandoli [21], Flandoli and Schmalfuss [26], Imkeller and Schmalfuss [27], and others. Due to the unbounded fluctuations in the systems caused by the white noise, the concept of pull-back global random attractor was introduced to capture the essential dynamics with possibly extremely wide fluctuations. This is significantly different from the deterministic case.

In the present paper, we prove the existence of a global random attractor for the infinite dimensional random dynamical system generated by the stochastic lattice differential equation (1.1). An interesting feature of this is that, even though the spatial domain is unbounded and the solution operator is not smoothing or compact, unlike parabolic type of partial differential equations on bounded domains, bounded sets of initial data converge, in the pullback sense, under the forward flow to a random compact invariant set. The noise involved here is multiplicative. The domain of attraction is the set of all tempered sets as used in [26] instead of all bounded deterministic sets.

It is worth mentioning that, although it is known that finite dimensional stochastic differential equations generate random dynamical systems (see Arnold [2] Chapter 1), this is not true in general for infinite dimensional equations. However, for particular kinds of noise it is possible to transform the stochastic differential equation into a random one so that it generates a random dynamical system. For general types of noise it is unknown whether this is possible.

In Section 2, we introduce basic concepts concerning random dynamical systems and global random attractors. In Section 3, we show that the stochastic lattice differential equation (1.1) generates a infinite dimensional random dynamical system. The existence of the global random attractor is given in Section 4 .

\section{Random Dynamical Systems}

In this section, we introduce basic concepts related to random dynamical systems and the concept of attractor, which are taken from [2] and [26].

Let $\left(H,\|\cdot\|_{H}\right)$ be a Hilbert space and $(\Omega, \mathcal{F}, \mathbb{P})$ be a probability space.

Definition 2.1. $\left(\Omega, \mathcal{F}, \mathbb{P},\left(\theta_{t}\right)_{t \in \mathbb{R}}\right)$ is called a metric dynamical system, if $\theta: \mathbb{R} \times \Omega \rightarrow \Omega$ is $(\mathcal{B}(\mathbb{R}) \times \mathcal{F}, \mathcal{F})$-measurable, $\theta_{0}$ is the identity on $\Omega, \theta_{s+t}=\theta_{t} \circ \theta_{s}$ for all $s, t \in \mathbb{R}$ and $\theta_{t} \mathbb{P}=\mathbb{P}$ for all $t \in \mathbb{R}$.

Definition 2.2. A stochastic process $(\varphi(t))_{t>0}$ is a continuous random dynamical system over $\left(\Omega, \mathcal{F}, \mathbb{P},\left(\theta_{t}\right)_{t \in \mathbb{R}}\right)$ if $\varphi$ is $(\mathcal{B}[0, \infty) \times \mathcal{F} \times \mathcal{B}(H), \mathcal{B}(H))$-measurable, and for all $\omega \in \Omega$

(D1) the mapping $\varphi(\cdot, \omega, \cdot):[0, \infty) \times H \rightarrow H$ is continuous,

(D2) $\varphi(0, \omega, \cdot)$ is the identity on $H$,

(D3) $\varphi(s+t, \omega, \cdot)=\varphi\left(t, \theta_{s} \omega, \cdot\right) \circ \varphi(s, \omega, \cdot) \quad$ for all $s, t \geq 0$ (cocycle property).

As we mentioned in the introduction, it is known that finite dimensional stochastic differential equations generate random dynamical systems (see Arnold [2] Chapter 1). This is not true in general for infinite dimensional equations. However, for particular kinds of noise, as will be our case, we can apply the following simple lemma to obtain a random dynamical system (see Caraballo et al. [9]). 
Lemma 2.3. Let $\psi$ be a random dynamical system. Suppose that the mapping $T: \Omega \times H \rightarrow H$ possesses the following properties: For fixed $\omega \in \Omega$, the mapping $T(\omega, \cdot)$ is a homeomorphism on $H$, and for fixed $x \in H$, the mappings $T(\cdot, x), T^{-1}(\cdot, x)$ are measurable. Then the mapping

$$
(t, \omega, x) \rightarrow \varphi(t, \omega, x):=T^{-1}\left(\theta_{t} \omega, \psi(t, \omega, T(\omega, x))\right)
$$

is a (conjugated) random dynamical system.

The measurability of $\varphi$ follows because of the properties of $T$. Later on we will transform our stochastic evolution equation containing a noisy term into an evolution equation without noise but with random coefficients.

Definition 2.4. A set $A \subset \Omega$ is called invariant with respect to $\left(\theta_{t}\right)_{t \in \mathbb{R}}$ if for all $t \in \mathbb{R}$ it holds

$$
\theta_{t}^{-1} A=A \text {. }
$$

Definition 2.5. A random set $A(\omega)$ is a multi-valued map $A: \Omega \rightarrow 2^{H} \backslash \emptyset$ such that, for every $x \in H$, the map $\omega \mapsto d(x, A(\omega))$ is measurable. It is said that the random set is bounded (resp. closed or compact) if $A(\omega)$ is bounded (resp. closed or compact) for almost all $\omega \in \Omega$.

Definition 2.6. A random bounded set $B(\omega) \subset H$ is called tempered with respect to $\left(\theta_{t}\right)_{t \in \mathbb{R}}$ if for a.e. $\omega \in \Omega$

$$
\lim _{t \rightarrow \infty} e^{-\beta t} d\left(B\left(\theta_{-t} \omega\right)\right)=0 \text { for all } \beta>0,
$$

where $d(B)=\sup _{x \in B}\|x\|_{H}$.

We consider a continuous random dynamical system $(\varphi(t))_{t \geq 0}$ over $\left(\Omega, \mathcal{F}, \mathbb{P},\left(\theta_{t}\right)_{t \in \mathbb{R}}\right)$ and $\mathcal{D}$ a collection of random subsets of $H$.

Definition 2.7. A random set $K$ is called an absorbing set in $\mathcal{D}$ if for all $B \in \mathcal{D}$ and a.e. $\omega \in \Omega$ there exists $t_{B}(\omega)>0$ such that

$$
\varphi\left(t, \theta_{-t} \omega, B\left(\theta_{-t} \omega\right)\right) \subset K(\omega) \text { for all } t \geq t_{B}(\omega) .
$$

Definition 2.8. $A$ random set $\mathcal{A}$ is called a global random $\mathcal{D}$ attractor (pullback $\mathcal{D}$ attractor) for $\varphi$ if the following hold:

(A1) $\mathcal{A}$ is a random compact set;

(A2) $\mathcal{A}$ is strictly invariant, i.e. for a.e. $\omega \in \Omega$ and all $t \geq 0$ one has $\varphi(t, \omega, \mathcal{A}(\omega))=\mathcal{A}\left(\theta_{t} \omega\right)$;

(A3) $\mathcal{A}$ attracts all sets in $\mathcal{D}$, i.e., for all $B \in \mathcal{D}$ and a.e. $\omega \in \Omega$ we have

$$
\lim _{t \rightarrow \infty} d\left(\varphi\left(t, \theta_{-t} \omega, B\left(\theta_{-t} \omega\right)\right), \mathcal{A}(\omega)\right)=0,
$$

where $d(X, Y)=\sup _{x \in X} \inf _{y \in Y}\|x-y\|_{H}$ is the Hausdorff semi-metric (here $X \subseteq H, Y \subseteq H$ ).

The collection $\mathcal{D}$ is called domain of attraction of $\mathcal{A}$.

Some possibilities for domains $\mathcal{D}$ of attraction used very often in the investigation of random attractors are the following:

1) the collection of all finite deterministic subsets of $H$ (in this case the random $\mathcal{D}$ attractor is called a point attractor);

2) the collection of all compact deterministic subsets of $H$ (in this case the random $\mathcal{D}$ attractor is called a set attractor); 
3) the collection of all tempered random subsets of $H$.

Examples of point attractors and set attractors can be found in [38], [19] and also in [2] (Theorem 9.3.3 pp. 484, Lemma 9.3.5 pp. 485). The results on random $\mathcal{D}$ attractors, where $\mathcal{D}$ is the collection of tempered random sets, can be found in [25], [26], [27].

The next proposition is an abstract result on the existence of global random attractor, which is a slight generalization of Theorem 3.5 on pg. 27 in [26] (see [5] for the proof). Although it may be possible to apply a more general result from Caraballo et al. [10], the following one will fit our purposes.

Proposition 2.9. Let $K \in \mathcal{D}$ be an absorbing set for the continuous random dynamical system $(\varphi(t))_{t \geq 0}$ which is closed, and which satisfies for a.e. $\omega \in \Omega$ the following asymptotic compactness condition: each sequence $x_{n} \in \varphi\left(t_{n}, \theta_{-t_{n}}, K\left(\theta_{-t_{n}} \omega\right)\right)$ with $t_{n} \rightarrow \infty$ has a convergent subsequence in $H$. Then the cocycle $\varphi$ has a unique global random attractor

$$
\mathcal{A}(\omega)=\bigcap_{\tau \geq t_{K}(\omega)} \overline{\bigcup_{t \geq \tau} \varphi\left(t, \theta_{-t} \omega, K\left(\theta_{-t} \omega\right)\right)} .
$$

\section{Stochastic Lattice Differential Equations}

We consider a stochastic lattice differential equation

$$
\frac{d u_{i}(t)}{d t}=\nu\left(u_{i-1}-2 u_{i}+u_{i+1}\right)-\lambda u_{i}-f_{i}\left(u_{i}\right)+g_{i}+\sum_{j=1}^{N} c_{j} u_{i} \circ \frac{d w_{j}(t)}{d t}, \quad i \in \mathbb{Z},
$$

where $u=\left(u_{i}\right)_{i \in \mathbb{Z}} \in \ell^{2}, \mathbb{Z}$ denotes the integer set, $\nu$ and $\lambda$ are positive constants, $f_{i}$ is a smooth function satisfying a dissipative condition, $g=\left(g_{i}\right)_{i \in \mathbb{Z}} \in \ell^{2}, c_{j} \in \mathbb{R}$, for $j=1, \ldots, N$, and $w_{j}$ are mutually independent two-sided Brownian motions on the same probability space $(\Omega, \mathcal{F}, \mathbb{P})$.

We note that equation (3.1) is interpreted as a system of integral equations

$$
\begin{gathered}
u_{i}(t)=u_{i}(0)+\int_{0}^{t}\left(\nu\left(u_{i-1}(s)-2 u_{i}(s)+u_{i+1}(s)\right)-\lambda u_{i}(s)-f_{i}\left(u_{i}(s)\right)+g_{i}\right) d s \\
+\int_{0}^{t} \sum_{j=1}^{N} c_{j} u_{i}(s) \circ d w_{j}(t), \quad i \in \mathbb{Z},
\end{gathered}
$$

where the stochastic integral is understood in the sense of Stratonovich.

Assumptions on the nonlinearity $\boldsymbol{f}_{\boldsymbol{i}}$ : Let $f_{i} \in \mathcal{C}^{1}(\mathbb{R})$ satisfy that $\sup _{i \in \mathbb{Z}}\left|f_{i}{ }^{\prime}(u)\right|$ is bounded for $u$ in bounded sets and

$$
f_{i}(x) x \geq 0 \quad \text { for all } x \in \mathbb{R} .
$$

We note that condition (3.3) implies $f_{i}(0)=0$. If $f_{i}(s)=\sum_{j=0}^{p} a_{j} s^{2 j+1}$ with $a_{j} \geq 0$ for each $j=0, \ldots, p$, then conditions (3.3) is satisfied. This kind of nonlinearity was considered in [11] and $[22]$. 
For convenience, we now formulate system (3.1) as a stochastic differential equation in $\ell^{2}$. Denote by $\|\cdot\|$ the norm in the space $\ell^{2}$, and by $B, B^{*}, C_{j}, j=1, \ldots, N$, and $A$ the linear operators from $\ell^{2}$ to $\ell^{2}$ defined as follows. For $u=\left(u_{i}\right)_{i \in \mathbb{Z}} \in \ell^{2}$,

$$
(B u)_{i}=u_{i+1}-u_{i}, \quad\left(B^{*} u\right)_{i}=u_{i-1}-u_{i}, \quad\left(C_{j} u\right)_{i}=c_{j} u_{i}
$$

and

$$
(A u)_{i}=-u_{i-1}+2 u_{i}-u_{i+1} \text { for each } i \in \mathbb{Z}
$$

Then we find that

$$
A=B B^{*}=B^{*} B
$$

and

$$
\left(B^{*} u, v\right)=(u, B v) \text { for all } u, v \in \ell^{2} .
$$

Therefore $(A u, u) \geq 0$ for all $u \in \ell^{2}$.

Let $\tilde{f}$ be the Nemytski operator associated with $f_{i}$, that is, for $u=\left(u_{i}\right)_{i \in \mathbb{Z}} \in \ell^{2}$, let $\tilde{f}(u)=$ $\left(f_{i}\left(u_{i}\right)\right)_{i \in \mathbb{Z}}$. Then we have

$$
\|\tilde{f}(u)\|^{2}=\sum_{i \in \mathbb{Z}}\left|f_{i}\left(u_{i}\right)\right|^{2}=\sum_{i \in \mathbb{Z}}\left|f_{i}\left(u_{i}\right)-f_{i}(0)\right|^{2}=\sum_{i \in \mathbb{Z}}\left|f_{i}^{\prime}\left(\xi_{i}\right)\right|^{2}\left|u_{i}\right|^{2},
$$

with $\xi_{i}=\tau_{i} u_{i}$ for some $\tau_{i} \in(0,1)$. Since

$$
\left|\xi_{i}\right| \leq\left|u_{i}\right| \leq\|u\|
$$

and $\sup _{i \in \mathbb{Z}}\left|f_{i}^{\prime}\right|$ is bounded on a bounded set, it follows that there exists a constant $\mu$ (depending on $\|u\|)$ such that

$$
\|\tilde{f}(u)\|^{2} \leq \mu \sum_{i \in \mathbb{Z}}\left|u_{i}\right|^{2}=\mu\|u\|^{2}
$$

which means $\tilde{f}(u) \in \ell^{2}$.

Similar to (3.4), one can see that $\tilde{f}$ is locally Lipschitz from $\ell^{2}$ to $\ell^{2}$, more precisely, for every bounded set $Y$ in $\ell^{2}$, there exists a constant $C_{Y}$ (depending only on $Y$ ) such that

$$
\|\tilde{f}(x)-\tilde{f}(y)\| \leq C_{Y}\|x-y\| \text { for all } x, y \in Y .
$$

In the sequel, when no confusion arises we identify $\tilde{f}$ with $f$.

The system (3.1) with initial values $u_{0} \equiv\left(u_{0, i}\right)_{i \in \mathbb{Z}} \in \ell^{2}$ may be rewritten as an equation in $\ell^{2}$ for $t \geq 0$ and $\omega \in \Omega$,

$$
u(t)=u_{0}+\int_{0}^{t}(-\nu A u(s)-\lambda u(s)-\tilde{f}(u(s))+g) d s+\sum_{j=1}^{N} \int_{0}^{t} C_{j} u(s) \circ d w_{j}(t) .
$$

To prove that this stochastic equation (3.5) generates a random dynamical system, we will transform it into a random differential equation in $\ell^{2}$. This can be done thanks to the special form of the stochastic term.

Before performing this transformation, we need to recall some properties of the Ornstein-Uhlenbeck processes. Let us start by describing a probability space $(\Omega, \mathcal{F}, \mathbb{P})$ which will be useful for our analysis. Consider

$$
\Omega=\{\omega \in \mathcal{C}(\mathbb{R}, \mathbb{R}): \omega(0)=0\}=C_{0}(\mathbb{R}, \mathbb{R})
$$


endowed with the compact open topology (see [2], Appendix A.2 and A.3), $\mathbb{P}$ is the corresponding Wiener measure and $\mathcal{F}_{0}$ is the Borel $\sigma$-algebra on $\Omega$. Let

$$
\theta_{t} \omega(\cdot)=\omega(\cdot+t)-\omega(t), \quad t \in \mathbb{R},
$$

then $\left(\Omega, \mathcal{F}_{0}, \mathbb{P},\left(\theta_{t}\right)_{t \in \mathbb{R}}\right)$ is a metric dynamical system. Let $\mathcal{F}$ be the $\mathbb{P}$-completion of $\mathcal{F}_{0}$ and let

$$
\mathcal{F}_{t}:=\bigvee_{s \leq t} \mathcal{F}_{s}^{t}, \quad t \in \mathbb{R}
$$

with

$$
\mathcal{F}_{s}^{t}=\sigma\left\{w\left(\tau_{2}\right)-w\left(\tau_{1}\right): s \leq \tau_{1} \leq \tau_{2} \leq t\right\} \bigvee \mathcal{N}
$$

where $\sigma\left\{w\left(\tau_{2}\right)-w\left(\tau_{1}\right): s \leq \tau_{1} \leq \tau_{2} \leq t\right\}$ is the smallest $\sigma$-algebra generated by the random variable $w\left(\tau_{2}\right)-w\left(\tau_{1}\right)$ for all $\tau_{1}, \tau_{2}$ such that $s \leq \tau_{1} \leq \tau_{2} \leq t$ and $\mathcal{N}$ are the null sets of $\mathcal{F}$.

Note that $\theta_{\tau}^{-1} \mathcal{F}_{s}^{t}=\mathcal{F}_{s+\tau}^{t+\tau}$, so $\left(\Omega, \mathcal{F}_{0}, \mathbb{P},\left(\theta_{t}\right)_{t \in \mathbb{R}},\left(\mathcal{F}_{s}^{t}\right)_{s \leq t}\right)$ is a filtered dynamical system (see [2], pp. 72,91 and 546 for more details).

Let us consider the one-dimensional stochastic differential equation

$$
d z=-\alpha z d t+d w(t)
$$

for $\alpha>0$. This equation has a random fixed point in the sense of random dynamical systems generating a stationary solution known as the stationary Ornstein-Uhlenbeck process (see Caraballo et al. [9] for more details). In fact, we have

Lemma 3.1. (Caraballo et al. [9]) There exists a $\left\{\theta_{t}\right\}_{t \in \mathbb{R}}$-invariant subset $\bar{\Omega} \in \mathcal{F}$ of $\Omega=C_{0}(\mathbb{R}, \mathbb{R})$ of full measure such that

$$
\lim _{t \rightarrow \pm \infty} \frac{|\omega(t)|}{t}=0 \quad \text { for } \omega \in \bar{\Omega}
$$

and, for such $\omega$, the random variable given by

$$
z^{*}(\omega):=-\alpha \int_{-\infty}^{0} e^{\alpha \tau} \omega(\tau) d \tau
$$

is well defined. Moreover, for $\omega \in \bar{\Omega}$, the mapping

$$
\begin{aligned}
(t, \omega) \rightarrow z^{*}\left(\theta_{t} \omega\right) & =-\alpha \int_{-\infty}^{0} e^{\alpha \tau} \theta_{t} \omega(\tau) d \tau \\
& =-\alpha \int_{-\infty}^{0} e^{\alpha \tau} \omega(t+\tau) d \tau+\omega(t)
\end{aligned}
$$

is a stationary solution of (3.7) with continuous trajectories. In addition, for $\omega \in \bar{\Omega}$

$$
\begin{aligned}
& \lim _{t \rightarrow \pm \infty} \frac{\left|z^{*}\left(\theta_{t} \omega\right)\right|}{|t|}=0, \quad \lim _{t \rightarrow \pm \infty} \frac{1}{t} \int_{0}^{t} z^{*}\left(\theta_{\tau} \omega\right) d \tau=0, \\
& \lim _{t \rightarrow \pm \infty} \frac{1}{t} \int_{0}^{t}\left|z^{*}\left(\theta_{\tau} \omega\right)\right| d \tau=\mathbb{E}\left|z^{*}\right|<\infty .
\end{aligned}
$$

Remark 3.2. We now consider $\theta$ defined in (3.6) on $\bar{\Omega}$ instead of $\Omega$. This mapping possesses the same properties as the original one if we choose for $\mathcal{F}$ the trace $\sigma$-algebra with respect to $\bar{\Omega}$ denoted also by $\mathcal{F}$. 
Let us consider $\alpha=1$ and denote by $z_{j}^{*}$ its associated Ornstein-Uhlenbeck process corresponding to (3.7) with $w_{j}$ instead of $w$.

Then for any $j=1, \ldots, N$ we have a stationary Ornstein-Uhlenbeck process generated by a random variable $z_{j}^{*}(\omega)$ on $\bar{\Omega}_{j}$ with properties formulated in Lemma 3.1 defined on the metric dynamical system $\left(\bar{\Omega}_{j}, \mathcal{F}_{j}, \mathbb{P}_{j}, \theta\right)$. We set

$$
(\Omega, \mathcal{F}, \mathbb{P}, \theta),
$$

where

$$
\Omega=\bar{\Omega}_{1} \times \cdots \times \bar{\Omega}_{N}, \quad \mathcal{F}=\bigotimes_{i=1}^{N} \mathcal{F}_{i}, \quad \mathbb{P}=\mathbb{P}_{1} \times \mathbb{P}_{2} \times \cdots \times \mathbb{P}_{N},
$$

and $\theta$ is the flow of Wiener shifts.

Now, let us note that operator $C_{j}$ generates a strongly continuous semigroup (in fact, a uniformly continuous group) of operators $S_{C j}(t)$. More precisely, $S_{C j}(t)$ is given by

$$
S_{C_{j}}(t) u=e^{c_{j} t} u, \quad \text { for } u \in \ell^{2} .
$$

Then we denote

$$
T(\omega):=S_{C_{1}}\left(z_{1}^{*}(\omega)\right) \circ \ldots \circ S_{C_{N}}\left(z_{N}^{*}(\omega)\right)=e^{\sum_{j=1}^{N} c_{j} z_{j}^{*}(\omega)} I d_{\ell^{2}}
$$

which is clearly a homeomorphism in $H=\ell^{2}$. The inverse operator is well defined by

$$
T^{-1}(\omega):=S_{C_{N}}\left(-z_{N}^{*}(\omega)\right) \circ \ldots \circ S_{C_{1}}\left(-z_{1}^{*}(\omega)\right)=e^{-\sum_{j=1}^{N} c_{j} z_{j}^{*}(\omega)} I d_{\ell^{2}} .
$$

From simplicity, let us denote $\delta(\omega)=\sum_{j=1}^{N} c_{j} z_{j}^{*}(\omega)$. It easily follows that $\left\|T^{-1}\left(\theta_{t} \omega\right)\right\|$ has subexponential growth as $t \rightarrow \pm \infty$ for any $\omega \in \Omega$. Hence $\left\|T^{-1}\right\|$ is tempered. According to Remark 3.2 we can change our metric dynamical system with respect to $\bar{\Omega}$. However the new metric dynamical system will be denoted by the same symbols $(\Omega, \mathcal{F}, \mathbb{P}, \theta)$.

We now argue in a heuristic informal way. Let us consider the change of variable

$$
v(t)=T^{-1}\left(\theta_{t} \omega\right) u(t)=e^{-\delta\left(\theta_{t} \omega\right)} u(t),
$$

where $u$ is a solution to (3.5). Then,

$$
\begin{aligned}
d v(t) & =e^{-\delta\left(\theta_{t} \omega\right)} d u(t)-\sum_{j=1}^{N} c_{j} e^{-\delta\left(\theta_{t} \omega\right)} u(t) \circ d z_{j}^{*}\left(\theta_{t} \omega\right) \\
& =e^{-\delta\left(\theta_{t} \omega\right)}\left(-\nu A u(t)-\lambda u(t)-\tilde{f}(u(t))+g+\delta\left(\theta_{t} \omega\right) u(t)\right) d t \\
& =\left(-\nu A v(t)-\lambda v(t)-e^{-\delta\left(\theta_{t} \omega\right)} \tilde{f}\left(e^{\delta\left(\theta_{t} \omega\right)} v(t)\right)+e^{-\delta\left(\theta_{t} \omega\right)} g+\delta\left(\theta_{t} \omega\right) v(t)\right) d t .
\end{aligned}
$$

So we can consider the following evolution equation with random coefficients but without white noise

$$
\frac{d v}{d t}=-\nu A v-\left(\lambda-\delta\left(\theta_{t} \omega\right)\right) v-e^{-\delta\left(\theta_{t} \omega\right)} \tilde{f}\left(e^{\delta\left(\theta_{t} \omega\right)} v\right)+e^{-\delta\left(\theta_{t} \omega\right)} g
$$

and initial condition $v(0)=v_{0} \in H$.

Now we establish the following result. 
Theorem 3.3. Let $T>0$ and $v_{0} \in H$ be fixed. Then the following properties hold:

(1) For every $\omega \in \Omega$, equation (3.10) admits a unique solution $v\left(\cdot, \omega, v_{0}\right) \in \mathcal{C}\left([0, T], \ell^{2}\right)$,

(2) The solution $v$ of (3.10) depends continuously on the initial data $v_{0}$, i.e., for each $\omega \in \Omega$ the mapping $v_{0} \in \ell^{2} \mapsto v\left(\cdot, \omega, v_{0}\right) \in \mathcal{C}\left([0, T], \ell^{2}\right)$ is continuous.

Proof. (1) For any fixed $T>0$ and $\omega \in \Omega$, and thanks to standard arguments (notice that the mapping $F(t, v)=e^{-\delta\left(\theta_{t} \omega\right)} f\left(e^{\delta\left(\theta_{t} \omega\right)} v\right)$ is locally Lipschitz with respect to its second variable and the Lipschitz constant is uniformly bounded in $[0, T])(3.10)$ possesses a local solution $v\left(\cdot ; \omega, v_{0}\right) \in$ $\mathcal{C}\left(\left[0, T_{\max }\right), \ell^{2}\right)$, where $\left[0, T_{\max }\right)$ is the maximal interval of existence of the solution of $(3.10)$. We prove now that this local solution is a global one. From (3.10) it follows that

$$
\begin{aligned}
\frac{d}{d t}\|v(t)\|^{2}= & 2\left(-\nu(A v, v)-\lambda\|v\|^{2}-\left(e^{-\delta\left(\theta_{t} \omega\right)} \tilde{f}\left(e^{\delta\left(\theta_{t} \omega\right)} v\right), v\right)+\delta\left(\theta_{t} \omega\right)\|v\|^{2}\right) \\
& +2\left(e^{-\delta\left(\theta_{t} \omega\right)} g, v\right) \\
\leq & -2 \lambda\|v\|^{2}+2 \delta\left(\theta_{t} \omega\right)\|v\|^{2}+\lambda\|v\|^{2}+\frac{1}{\lambda} e^{-2 \delta\left(\theta_{t} \omega\right)}\|g\|^{2} \\
\leq & \left(-\lambda+2 \delta\left(\theta_{t} \omega\right)\right)\|v\|^{2}+\frac{1}{\lambda} e^{-2 \delta\left(\theta_{t} \omega\right)}\|g\|^{2}
\end{aligned}
$$

whence

$$
\begin{aligned}
\|v(t)\|^{2} \leq & e^{-\lambda t+2 \int_{0}^{t} \delta\left(\theta_{s} \omega\right) d s}\left\|v_{0}\right\|^{2} \\
& +\frac{\|g\|^{2}}{\lambda} e^{-\lambda t+2 \int_{0}^{t} \delta\left(\theta_{s} \omega\right) d s} \int_{0}^{t} e^{-2 \delta\left(\theta_{s} \omega\right)+\lambda s-2 \int_{0}^{s} \delta\left(\theta_{r} \omega\right) d r} d s .
\end{aligned}
$$

If we denote $\beta(\omega)=\max _{t \in[0, T]}\left(\frac{\|g\|^{2}}{\lambda} e^{-\lambda t+2 \int_{0}^{t} \delta\left(\theta_{s} \omega\right) d s} \int_{0}^{t} e^{-2 \delta\left(\theta_{s} \omega\right)+\lambda s-2 \int_{0}^{s} \delta\left(\theta_{r} \omega\right) d r} d s\right)$ and $\alpha(\omega)=$ $2 \int_{0}^{T}\left|\delta\left(\theta_{s} \omega\right)\right| d s$, then we have that

$$
\|v(t)\|^{2} \leq\left\|v_{0}\right\|^{2} e^{\alpha(\omega)}+\beta(\omega)
$$

which implies that the solution $v$ is defined in any interval $[0, T]$.

(2) Let $u_{0}, v_{0} \in \ell^{2}$ and $X(t):=v\left(t, \omega, u_{0}\right), Y(t):=v\left(t, \omega, v_{0}\right)$ the corresponding solutions of (3.10). Then, if we denote $Z(t)=X(t)-Y(t)$ we have

$$
\frac{d Z(t)}{d t}=-\nu A Z(t)-\left(\lambda-\delta\left(\theta_{t} \omega\right)\right) Z(t)-e^{-\delta\left(\theta_{t} \omega\right)}\left(\tilde{f}\left(e^{\delta\left(\theta_{t} \omega\right)} X(t)\right)-\tilde{f}\left(e^{\delta\left(\theta_{t} \omega\right)} Y(t)\right)\right)
$$

and,

$$
\begin{aligned}
\frac{d}{d t}\|Z(t)\|^{2} & =2\left(-\nu(A Z, Z)-\lambda\|Z\|^{2}-e^{-\delta\left(\theta_{t} \omega\right)}\left(\tilde{f}\left(e^{\delta\left(\theta_{t} \omega\right)} X(t)\right)-\tilde{f}\left(e^{\delta\left(\theta_{t} \omega\right)} Y(t)\right), Z\right)\right) \\
& +2 \delta\left(\theta_{t} \omega\right)\|Z\|^{2} \\
& \leq 2\left(e^{-\delta\left(\theta_{t} \omega\right)}\left\|\tilde{f}\left(e^{\delta\left(\theta_{t} \omega\right)} X(t)\right)-\tilde{f}\left(e^{\delta\left(\theta_{t} \omega\right)} Y(t)\right)\right\|\|Z\|+\delta\left(\theta_{t} \omega\right)\|Z\|^{2}\right) \\
& \leq 2\left(L\|Z\|^{2}+\delta\left(\theta_{t} \omega\right)\|Z\|^{2}\right) \\
& \leq \gamma\|Z\|^{2},
\end{aligned}
$$

where $\gamma=2\left(L+\max _{t \in[0, T]}\left|\delta\left(\theta_{t} \omega\right)\right|\right)$, and $L$ denotes the Lipschitz constant of $f$ corresponding to a bounded set where $X$ and $Y$ belong to (see (3.12)). 
Now, by standard computations, we obtain that

$$
\|Z(t)\|^{2} \leq e^{\gamma t}|| Z(0) \|^{2}
$$

and, consequently,

$$
\sup _{t \in[0, T]}\|X(t)-Y(t)\|^{2} \leq e^{\gamma T}\left\|u_{0}-v_{0}\right\|^{2} .
$$

If $u_{0}=v_{0}$, then the above inequality shows the uniqueness and continuous dependence on the initial data of the solution of (3.10). So, the properties (1) and (2) of this theorem hold.

Theorem 3.4. Equation (3.10) generates a continuous random dynamical system $(\psi(t))_{t \geq 0}$ over $\left(\Omega, \mathcal{F}_{0}, P,\left(\theta_{t}\right)_{t \in \mathbb{R}}\right)$, where

$$
\psi\left(t, \omega, u_{0}\right)=v\left(t, \omega, u_{0}\right) \quad \text { for } u_{0} \in \ell^{2}, t \geq 0 \text { and for all } \omega \in \Omega .
$$

Moreover, if we define $\varphi$ by

$$
\varphi\left(t, \omega, u_{0}\right)=T\left(\theta_{t} \omega\right) \psi\left(t, \omega, T^{-1}(\omega) u_{0}\right) \text { for } u_{0} \in \ell^{2}, t \geq 0 \text { and for all } \omega \in \Omega,
$$

then $\varphi$ is another random dynamical system for which the process

$$
(\omega, t) \rightarrow \varphi\left(t, \omega, u_{0}\right)
$$

solves (3.5) for any initial condition $u_{0} \in \ell^{2}$.

Proof. The fact that $\psi$ is a continuous random dynamical system follows from Theorem 3.3. The measurability of $\varphi$ follows by the properties of $T$. To prove that $\varphi$ and $\psi$ are conjugated random dynamical systems, we will use the chain rule. Then, omitting the arguments for simplicity, we have that

$$
\begin{aligned}
d(T \psi) & =T d \psi+\sum_{j=1}^{N} c_{j} T \psi \circ d z_{j}^{*} \\
& =T\left(-\nu A \psi-(\lambda-\delta) \psi-T^{-1} \tilde{f}(T \psi)+T^{-1} g\right) d t-\delta T \psi d t+\sum_{j=1}^{N} c_{j} T \psi \circ d w_{j} \\
& =(-\nu A(T \psi)-\lambda T \psi-\tilde{f}(T \psi)+g) d t+\sum_{j=1}^{N} c_{j} T \psi \circ d w_{j},
\end{aligned}
$$

and the proof is complete.

\section{Existence of Global Random Attractors}

In this section, we prove the existence of a global random attractor for the random lattice dynamical system generated by equation (3.1). Our main result is

Theorem 4.1. The random lattice dynamical system $\varphi$ generated by Eq. (3.5) has a unique global random attractor.

To prove this theorem we will use Proposition 2.9. In order to prove that our lattice random dynamical system $\varphi$ satisfies the assumptions in the proposition above, we will proceed in the following way. We will prove first that there exists an absorbing set for $\psi$ in $\mathcal{D}$. Next we will construct a corresponding closed absorbing one in $\mathcal{D}$ for its conjugated lattice random dynamical system $\varphi$. Finally, we will prove the asymptotic compactness. 


\subsection{Existence of the closed tempered random absorbing set.}

We need to prove that there exists a closed random tempered set $K \in \mathcal{D}$ such that for all $B \in \mathcal{D}$ and a.e. $\omega \in \Omega$, there exists $T_{B, \omega}>0$ such that

$$
\varphi\left(t, \theta_{-t} \omega, B\left(\theta_{-t} \omega\right)\right) \subset K(\omega), \quad \text { for all } t \geq T_{B, \omega} .
$$

Let us start with $v(t)=\psi\left(t, \omega, u_{0}\right)$. Then, by arguing as in (3.11) we obtain

$$
\begin{aligned}
\|v(t)\|^{2} \leq & e^{-\lambda t+2 \int_{0}^{t} \delta\left(\theta_{s} \omega\right) d s}\left\|u_{0}\right\|^{2} \\
& +\frac{\|g\|^{2}}{\lambda} e^{-\lambda t+2 \int_{0}^{t} \delta\left(\theta_{s} \omega\right) d s} \int_{0}^{t} e^{-2 \delta\left(\theta_{s} \omega\right)+\lambda s-2 \int_{0}^{s} \delta\left(\theta_{r} \omega\right) d r} d s .
\end{aligned}
$$

Let us now substitute $\omega$ by $\theta_{-t} \omega$ and $u_{0}$ by $e^{-\delta\left(\theta_{-t} \omega\right)} u_{0}$ in the expression of $\psi$, we then have that

$$
\begin{aligned}
\left\|\psi\left(t, \theta_{-t} \omega, e^{-\delta\left(\theta_{-t} \omega\right)} u_{0}\right)\right\|^{2} \leq & e^{-\lambda t+2 \int_{0}^{t} \delta\left(\theta_{s-t} \omega\right) d s}\left\|e^{-\delta\left(\theta_{-t} \omega\right)} u_{0}\right\|^{2} \\
& +\frac{\|g\|^{2}}{\lambda} e^{-\lambda t+\int_{0}^{t} \delta\left(\theta_{s-t} \omega\right) d s} \int_{0}^{t} e^{-2 \delta\left(\theta_{s-t} \omega\right)+\lambda s-2 \int_{0}^{s} \delta\left(\theta_{r-t} \omega\right) d r} d s \\
\leq & e^{-\lambda t-\delta\left(\theta_{-t} \omega\right)+2 \int_{-t}^{0} \delta\left(\theta_{s} \omega\right) d s}\left\|u_{0}\right\|^{2} \\
& +\frac{\|g\|^{2}}{\lambda} \int_{0}^{t} e^{-2 \delta\left(\theta_{s-t} \omega\right)-\lambda(t-s)+2 \int_{s}^{t} \delta\left(\theta_{r-t} \omega\right) d r} d s \\
\leq & e^{-\lambda t-\delta\left(\theta_{-t} \omega\right)+2 \int_{-t}^{0} \delta\left(\theta_{s} \omega\right) d s}\left\|u_{0}\right\|^{2} \\
& +\frac{\|g\|^{2}}{\lambda} \int_{-t}^{0} e^{-2 \delta\left(\theta_{s} \omega\right)-\lambda s+2 \int_{s}^{0} \delta\left(\theta_{r} \omega\right) d r} d s \\
\leq & e^{-\lambda t-\delta\left(\theta_{-t} \omega\right)+2 \int_{-t}^{0} \delta\left(\theta_{s} \omega\right) d s}\left\|u_{0}\right\|^{2} \\
& +\frac{\|g\|^{2}}{\lambda} \int_{-\infty}^{0} e^{-2 \delta\left(\theta_{s} \omega\right)-\lambda s+2 \int_{s}^{0} \delta\left(\theta_{r} \omega\right) d r} d s .
\end{aligned}
$$

Notice that, thanks to the properties of the Ornstein-Uhlenbeck process $z^{*}$, it follows that

$$
\int_{-\infty}^{0} e^{-2 \delta\left(\theta_{s} \omega\right)-\lambda s+2 \int_{s}^{0} \delta\left(\theta_{r} \omega\right) d r} d s<+\infty
$$

Taking into account that for any $u_{0} \in B\left(\theta_{-t} \omega\right)$

$$
\varphi\left(t, \theta_{-t} \omega, u_{0}\right)=e^{\delta(\omega)} \psi\left(t, \theta_{-t} \omega, e^{-\delta\left(\theta_{-t} \omega\right)} u_{0}\right)
$$

then

$$
\begin{aligned}
\left\|\varphi\left(t, \theta_{-t} \omega, u_{0}\right)\right\|^{2} \leq & e^{\delta(\omega)} e^{-\lambda t-\delta\left(\theta_{-t} \omega\right)+2 \int_{-t}^{0} \delta\left(\theta_{s} \omega\right) d s} \mathrm{~d}\left(B\left(\theta_{-t} \omega\right)\right)^{2} \\
& +e^{\delta(\omega)} \frac{\|g\|^{2}}{\lambda} \int_{-\infty}^{0} e^{-2 \delta\left(\theta_{s} \omega\right)+\lambda s+2 \int_{s}^{0} \delta\left(\theta_{r} \omega\right) d r} d s
\end{aligned}
$$

Denoting by

$$
R^{2}(\omega)=2 e^{\delta(\omega)} \frac{\|g\|^{2}}{\lambda} \int_{-\infty}^{0} e^{-2 \delta\left(\theta_{s} \omega\right)+\lambda s+2 \int_{s}^{0} \delta\left(\theta_{r} \omega\right) d r} d s
$$


and noticing that

$$
\lim _{t \rightarrow+\infty} e^{\delta(\omega)} e^{-\lambda t-\delta\left(\theta_{-t} \omega\right)+2 \int_{-t}^{0} \delta\left(\theta_{s} \omega\right) d s} \mathrm{~d}\left(B\left(\theta_{-t} \omega\right)\right)^{2}=0,
$$

it follows that

$$
K(\omega)=\overline{B_{\ell^{2}}(0, R(\omega))}
$$

is an absorbing closed random set. We will now prove that $K \in \mathcal{D}$. To this end, we only have to check that

$$
\lim _{t \rightarrow+\infty} e^{-\beta t} R\left(\theta_{-t} \omega\right)=0
$$

Indeed, observe that

$$
\begin{aligned}
e^{-\beta t} R^{2}\left(\theta_{-t} \omega\right) & =2 e^{-\beta t} e^{\delta\left(\theta_{-t} \omega\right)} \frac{\|g\|^{2}}{\lambda} \int_{-\infty}^{0} e^{-2 \delta\left(\theta_{s-t} \omega\right)+\lambda s+2 \int_{s}^{0} \delta\left(\theta_{r-t} \omega\right) d r} d s \\
& =2 \underbrace{e^{-\beta t} e^{\delta\left(\theta_{-t} \omega\right)}}_{\longrightarrow 0 \text { as } t \rightarrow+\infty} \frac{\|g\|^{2}}{\lambda} \underbrace{\int_{-\infty}^{-t} e^{-2 \delta\left(\theta_{s} \omega\right)+\lambda(s+t)+2 \int_{s}^{-t} \delta\left(\theta_{r} \omega\right) d r} d s}_{\text {as } t \rightarrow+\infty} .
\end{aligned}
$$

\subsection{Asymptotic compactness.}

To prove the asymptotic compactness for the random dynamical system $\varphi$, we first prove the following lemma.

Lemma 4.2. Let $u_{0}(\omega) \in K(\omega)$, the absorbing set given by (4.3). Then for every $\epsilon>0$, there exist $T(\epsilon, \omega)>0$ and $N(\epsilon, \omega)>0$ such that the solution $\varphi$ of equation (3.1) satisfies

$$
\sum_{|i| \geq N(\epsilon, \omega)}\left|\varphi_{i}\left(t, \theta_{-t} \omega, u_{0}\left(\theta_{-t} \omega\right)\right)\right|^{2} \leq \epsilon, \text { for all } t \geq T(\epsilon, \omega) .
$$

Proof. Choose a smooth function $\rho$ such that $0 \leq \rho(s) \leq 1$ for $s \in \mathbb{R}^{+}$, and

$$
\rho(s)=0 \text { for } 0 \leq s \leq 1 ; \quad \rho(s)=1 \text { for } s \geq 2 .
$$

Then there exists a constant $C$ such that $\left|\rho^{\prime}(s)\right| \leq C$ for $s \in \mathbb{R}^{+}$.

We first consider the random equation (3.10). Let $k$ be a fixed integer which will be specified later, and set $x=\left(x_{i}\right)_{i \in \mathbb{Z}}$ with $x_{i}=\theta\left(\frac{|i|}{k}\right) \psi_{i}$. Then taking the inner product of equation (3.10) with $x$ in $l^{2}$, we get

$$
\begin{aligned}
\frac{d}{d t} \sum_{i \in \mathbb{Z}} \rho\left(\frac{|i|}{k}\right)\left|\psi_{i}\right|^{2}= & -2 \nu(A \psi, x)-2\left(\lambda-\delta\left(\theta_{t} \omega\right)\right) \sum_{i \in \mathbb{Z}} \rho\left(\frac{|i|}{k}\right)\left|\psi_{i}\right|^{2} \\
& -2 e^{-\delta\left(\theta_{t} \omega\right)} \sum_{i \in \mathbb{Z}} \rho\left(\frac{|i|}{k}\right) f_{i}\left(e^{\delta\left(\theta_{t} \omega\right)} \psi_{i}\right) \psi_{i}+2 e^{-\delta\left(\theta_{t} \omega\right)} \sum_{i \in \mathbb{Z}} \rho\left(\frac{|i|}{k}\right) g_{i} \psi_{i} .
\end{aligned}
$$


We now estimate the terms in (4.4) as follows. First we have

$$
\begin{aligned}
& (A \psi, x)=(B \psi, B x) \\
& =\sum_{i \in \mathbb{Z}}\left(\psi_{i+1}-\psi_{i}\right)\left(x_{i+1}-x_{i}\right) \\
& =\sum_{i \in \mathbb{Z}}\left(\psi_{i+1}-\psi_{i}\right)\left[\left(\rho\left(\frac{|i+1|}{k}\right)-\rho\left(\frac{|i|}{k}\right)\right) \psi_{i+1}+\rho\left(\frac{|i|}{k}\right)\left(\psi_{i+1}-\psi_{i}\right)\right] \\
& =\sum_{i \in \mathbb{Z}}\left(\rho\left(\frac{|i+1|}{k}\right)-\rho\left(\frac{|i|}{k}\right)\right)\left(\psi_{i+1}-\psi_{i}\right) \psi_{i+1}+\sum_{i \in \mathbb{Z}} \rho\left(\frac{|i|}{k}\right)\left(\psi_{i+1}-\psi_{i}\right)^{2} \\
& \geq \sum_{i}\left(\rho\left(\frac{|i+1|}{k}\right)-\rho\left(\frac{|i|}{k}\right)\right)\left(\psi_{i+1}-\psi_{i}\right) \psi_{i+1} .
\end{aligned}
$$

By the property of the cut-off function $\rho$, we estimate

$$
\begin{aligned}
& \left|\sum_{i \in \mathbb{Z}}\left(\rho\left(\frac{|i+1|}{k}\right)-\rho\left(\frac{|i|}{k}\right)\right)\left(\psi_{i+1}-\psi_{i}\right) \psi_{i+1}\right| \\
& \leq \sum_{i \in \mathbb{Z}} \frac{\left|\rho^{\prime}\left(\xi_{i}\right)\right|}{k}\left|\psi_{i+1}-\psi_{i}\right|\left|\psi_{i+1}\right| \\
& \leq \frac{C}{k} \sum_{i}\left(\left|\psi_{i+1}\right|^{2}+\left|\psi_{i}\right|\left|\psi_{i+1}\right|\right) \leq \frac{2 C}{k}\|\psi\|^{2},
\end{aligned}
$$

which yields that

$$
(B \psi, B x) \geq \frac{-2 C\|\psi\|^{2}}{k} .
$$

Using condition (3.3), we have

$$
-2 e^{\delta\left(\theta_{t} \omega\right)} \sum_{i \in \mathbb{Z}} \rho\left(\frac{|i|}{k}\right) f_{i}\left(e^{\delta\left(\theta_{t} \omega\right)} \psi_{i}\right) \psi_{i} \leq 0 .
$$

For the last term in (4.4), we have

$$
\begin{aligned}
& \sum_{i \in \mathbb{Z}} \rho\left(\frac{|i|}{k}\right) g_{i} \psi_{i}=\sum_{|i| \geq k} \rho\left(\frac{|i|}{k}\right) g_{i} \psi_{i} \\
& \leq \frac{1}{8} \lambda \sum_{|i| \geq k} \rho\left(\frac{|i|}{k}\right)\left|\psi_{i}\right|^{2}+\frac{2}{\lambda} \sum_{|i| \geq k}\left|g_{i}\right|^{2} .
\end{aligned}
$$

Then, from (4.4) - (4.6) it follows that,

$$
\begin{aligned}
& \frac{d}{d t} \sum_{i \in \mathbb{Z}} \rho\left(\frac{|i|}{k}\right)\left|\psi_{i}\right|^{2}+\left(\lambda-2 \delta\left(\theta_{t} \omega\right)\right) \sum_{i \in \mathbb{Z}} \rho\left(\frac{|i|}{k}\right)\left|\psi_{i}\right|^{2} \\
& \leq \frac{4 \nu C}{k}\left\|\psi\left(t, \omega, e^{-\delta(\omega)} u_{0}\right)\right\|^{2}+\frac{4}{\lambda} e^{-\delta\left(\theta_{t} \omega\right)} \sum_{|i| \geq k}\left|g_{i}\right|^{2} .
\end{aligned}
$$


By using Gronwall's lemma, we have that for $t \geq T_{K}=T_{K}(\omega)$,

$$
\begin{aligned}
& \sum_{i \in \mathbb{Z}} \rho\left(\frac{|i|}{k}\right)\left|\psi_{i}\left(t, \omega, e^{-\delta(\omega)} u_{0}(\omega)\right)\right|^{2} \\
& \leq e^{-\lambda\left(t-T_{K}\right)+2 \int_{T_{k}}^{t} \delta\left(\theta_{s} \omega\right) d s} \sum_{i \in \mathbb{Z}} \rho\left(\frac{|i|}{k}\right)\left|\psi_{i}\left(T_{K}, \omega, e^{-\delta(\omega)} u_{0}(\omega)\right)\right|^{2} \\
& \quad+\left.\frac{4 \nu C}{k} \int_{T_{K}}^{t} e^{-\lambda(t-\tau)+2 \int_{\tau}^{t} \delta\left(\theta_{s}\right) d s}|| \psi\left(\tau, \omega, e^{-\delta(\omega)} u_{0}\right)\right|^{2} d \tau \\
& \quad+\frac{4}{\lambda}\left(\sum_{|i| \geq k}\left|g_{i}\right|^{2}\right) \int_{T_{K}}^{t} e^{-\lambda(t-\tau)+2 \int_{\tau}^{t} \delta\left(\theta_{s}\right) d s-\delta\left(\theta_{\tau} \omega\right)} d \tau .
\end{aligned}
$$

Replace $\omega$ by $\theta_{-t} \omega$. We then estimate each terms on the right hand side of (4.7). From (4.1) with $t$ replaced by $T_{K}$ and $\omega$ by $\theta_{-t} \omega$, it follows that

$$
\begin{aligned}
& e^{-\lambda\left(t-T_{K}\right)+2 \int_{T_{K}}^{t} \delta\left(\theta_{s-t} \omega\right) d s} \sum_{i \in \mathbb{Z}} \rho\left(\frac{|i|}{k}\right)\left|\psi_{i}\left(T_{K}, \theta_{-t} \omega, e^{-\delta\left(\theta_{-t} \omega\right)} u_{0}\left(\theta_{-t} \omega\right)\right)\right|^{2} \\
& \leq e^{-\lambda t+2 \int_{0}^{t} \delta\left(\theta_{s-t} \omega\right) d s-\delta\left(\theta_{-t} \omega\right)}\left\|u_{0}\right\|^{2}+\frac{\|g\|^{2}}{\lambda} \int_{0}^{T_{K}} e^{-2 \delta\left(\theta_{s-t} \omega\right)-\lambda(t-s)+2 \int_{s}^{t} \delta\left(\theta_{r-t} \omega\right) d r} d s .
\end{aligned}
$$

Thus, using (3.8), there is a $T_{1}(\epsilon, \omega)>T_{K}(\omega)$ such that if $t>T_{1}(\epsilon, \omega)$, then

$$
e^{-\lambda\left(t-T_{K}\right)+2 \int_{T_{K}}^{t} \delta\left(\theta_{s-t} \omega\right) d s} \sum_{i \in \mathbb{Z}} \rho\left(\frac{|i|}{k}\right)\left|\psi_{i}\left(T_{K}, \theta_{-t} \omega, e^{-\delta\left(\theta_{-t} \omega\right)} u_{0}\left(\theta_{-t} \omega\right)\right)\right|^{2} \leq \frac{1}{3} \epsilon e^{-\delta(\omega)} .
$$

Next, we estimate

$$
\begin{aligned}
& \frac{4 \nu C}{k} \int_{T_{K}}^{t} e^{-\lambda(t-\tau)+2 \int_{\tau}^{t} \delta\left(\theta_{s-t} \omega\right) d s}\left\|\psi\left(\tau, \theta_{-t} \omega, e^{-\delta\left(\theta_{-t} \omega\right)} u_{0}\right)\right\|^{2} d \tau \\
& \leq \frac{4 \nu C}{k}\left\|u_{0}\right\|\left(t-T_{K}\right) e^{-\lambda t+2 \int_{0}^{t} \delta\left(\theta_{s-t} \omega\right) d s-\delta\left(\theta_{-t} \omega\right)} \\
& \quad+\frac{\|g\|}{\lambda} \int_{T_{K}}^{t} \int_{0}^{\tau} e^{-2 \delta\left(\theta_{s-t} \omega\right)-\lambda(t-s)+2 \int_{s}^{t} \delta\left(\theta_{r-t} \omega\right) d r} d s d \tau .
\end{aligned}
$$

Then, using (3.8), there exist $T_{2}(\epsilon, \omega)>T_{K}(\omega)$, and $N_{1}(\epsilon, \omega)>0$ such that if $t>T_{2}(\epsilon, \omega)$ and $k>N_{1}(\epsilon, \omega)$, then

$$
\frac{4 \nu C}{k} \int_{T_{K}}^{t} e^{-\lambda(t-\tau)+2 \int_{\tau}^{t} \delta\left(\theta_{s-t} \omega\right) d s}\left\|\psi\left(\tau, \theta_{-t} \omega, e^{-\delta\left(\theta_{-t} \omega\right)} u_{0}\right)\right\|^{2} d \tau \leq \frac{1}{3} \epsilon e^{-\delta(\omega)} .
$$

Since $g \in \ell^{2}$, by using (3.8), there exists $N_{2}(\epsilon, \omega)>0$ such that for $k>N_{2}(\epsilon, \omega)$

$$
\frac{4}{\lambda}\left(\sum_{|i| \geq k}\left|g_{i}\right|^{2}\right) \int_{T_{K}}^{t} e^{-\lambda(t-\tau)+2 \int_{\tau}^{t} \delta\left(\theta_{s-t} \omega\right) d s-\delta\left(\theta_{\tau-t} \omega\right)} d \tau \leq \frac{1}{3} \epsilon e^{-\delta(\omega)} .
$$


Therefore, by letting

$$
\begin{aligned}
& T(\epsilon, \omega)=\max \left\{T_{1}(\epsilon, \omega), T_{2}(\epsilon, \omega)\right\}, \\
& N(\epsilon, \omega)=\max \left\{N_{1}(\epsilon, \omega), N_{2}(\epsilon, \omega)\right\},
\end{aligned}
$$

we have for $t>T(\epsilon, \omega)$ and $k>N(\epsilon, \omega)$

$$
\sum_{|i| \geq 2 k}\left|\psi_{i}\left(t, \theta_{-t} \omega, e^{-\delta\left(\theta_{-t} \omega\right)} u_{0}\left(\theta_{-t} \omega\right)\right)\right|^{2} \leq \sum_{i \in \mathbb{Z}} \rho\left(\frac{|i|}{k}\right)\left|\psi_{i}\left(t, \theta_{-t} \omega, e^{-\delta\left(\theta_{-t} \omega\right)} u_{0}\left(\theta_{-t} \omega\right)\right)\right|^{2} \leq \epsilon e^{-\delta(\omega)}
$$

which, thanks to relation (3.13), implies that

$$
\sum_{|i| \geq N(\epsilon, \omega)}\left|\varphi_{i}\left(t, \theta_{-t} \omega, u_{0}\left(\theta_{-t} \omega\right)\right)\right|^{2} \leq \epsilon
$$

provided $N(\epsilon, \omega)$ is large enough. This completes the proof of the lemma.

We are now ready to show the asymptotic compactness of $K$.

Theorem 4.3. For $\omega \in \Omega$ the set $K(\omega)$ is asymptotically compact: each sequence

$$
p_{n} \in \varphi\left(t_{n}, \theta_{-t_{n}} \omega, K\left(\theta_{-t_{n}} \omega\right)\right)
$$

with $t_{n} \rightarrow \infty$ has a convergent subsequence in $\ell^{2}$.

Proof. Consider $\left(t_{n}\right)_{n \in \mathbb{N}}$ with $\lim _{n \rightarrow \infty} t_{n}=\infty$ and $p_{n} \in \varphi\left(t_{n}, \theta_{-t_{n}} \omega, K\left(\theta_{-t_{n}} \omega\right)\right)$. We consider $p_{n}=$ $\varphi\left(t_{n}, \theta_{-t_{n}} \omega, x_{n}\right)$, where $x_{n} \in \stackrel{n \rightarrow \infty}{K}\left(\theta_{-t_{n}} \omega\right)$. We show that $\left(\varphi\left(t_{n}, \theta_{-t_{n}} \omega, x_{n}\right)\right)_{n \in \mathbb{N}}$ has a convergent subsequence. Since $K(\omega)$ is a bounded absorbing set, for large $n, \varphi\left(t_{n}, \theta_{-t_{n}} \omega, x_{n}\right) \in K(\omega)$. Thus, there exists $v \in \ell^{2}$ and a subsequence of $\left\{\varphi\left(t_{n}, \theta_{-t_{n}} \omega, x_{n}\right)\right\}$ (still denoted by $\left\{\varphi\left(t_{n}, \theta_{-t_{n}} \omega, x_{n}\right)\right\}$ ) such that

$$
\left\{\varphi\left(t_{n}, \theta_{-t_{n}} \omega, x_{n}\right)\right\} \rightarrow v \quad \text { weakly in } \ell^{2} .
$$

Next, we show that the above weak convergence is actually strong convergence, i.e., for each $\epsilon>0$ there is $N^{*}(\epsilon, \omega)>0$ such that for $n \geq N^{*}(\epsilon, \omega)$

$$
\left\|\varphi\left(t_{n}, \theta_{-t_{n}} \omega, x_{n}\right)-v\right\| \leq \epsilon .
$$

By Lemma 4.2, there exist $N_{1}^{*}(\epsilon, \omega)>0$ and $K_{1}(\epsilon, \omega)>0$ such that for $n>N_{1}^{*}$

$$
\sum_{|i| \geq K_{1}(\epsilon, \omega)}\left|\varphi_{i}\left(t_{n}, \theta_{-t_{n}} \omega, x_{n}\right)\right|^{2} \leq \frac{1}{8} \epsilon^{2} .
$$

On the other hand, since $v \in \ell^{2}$, there exists $K_{2}(\epsilon)$ such that

$$
\sum_{|i| \geq K_{2}(\epsilon)}\left|v_{i}\right|^{2} \leq \frac{1}{8} \epsilon^{2}
$$

Letting $K(\epsilon, \omega)=\max \left\{K_{1}(\epsilon, \omega), K_{2}(\epsilon)\right\}$, by the weak convergence (4.15), we have for each $|i| \leq$ $K(\epsilon, \omega)$ as $n \rightarrow \infty$

$$
\varphi_{i}\left(t_{n}, \theta_{-t_{n}} \omega, x_{n}\right) \rightarrow v_{i}
$$


which implies that there exists $N_{2}^{*}(\epsilon, \omega)>0$ such that when $n \geq N_{2}^{*}(\epsilon, \omega)$

$$
\sum_{|i| \leq K(\epsilon)}\left|\varphi_{i}\left(t_{n}, \theta_{-t_{n}} \omega, x_{n}\right)-v_{i}\right|^{2} \leq \frac{1}{2} \epsilon^{2} .
$$

Let $N^{*}(\epsilon, \omega)=\max \left\{N_{1}^{*}(\epsilon, \omega), N_{2}^{*}(\epsilon, \omega)\right\}$. Then, from (4.16), (4.17), and (4.18) we obtain for $n \geq N^{*}(\epsilon, \omega)$

$$
\begin{aligned}
& \left\|\varphi\left(t_{n}, \theta_{-t_{n}} \omega, x_{n}\right)-v\right\|^{2}=\sum_{|i| \leq K(\epsilon)}\left|\varphi_{i}\left(t_{n}, \theta_{-t_{n}} \omega, x_{n}\right)-v_{i}\right|^{2}+\sum_{|i|>K(\epsilon)}\left|\varphi_{i}\left(t_{n}, \theta_{-t_{n}} \omega, x_{n}\right)-v_{i}\right|^{2} \\
& \leq \frac{1}{2} \epsilon^{2}+2 \sum_{|i|>K(\epsilon)}\left(\left|\varphi_{i}\left(t_{n}, \theta_{-t_{n}} \omega, x_{n}\right)\right|^{2}+\left|v_{i}\right|^{2}\right) \leq \epsilon^{2} .
\end{aligned}
$$

Hence, $\varphi_{i}\left(t_{n}, \theta_{-t_{n}} \omega, x_{n}\right)$ strongly converges to $v$. The proof is complete.

Thus, we have proved Theorem 4.1.

In summary, we studied a stochastic lattice differential equation with diffusive nearest neighbor interaction, a dissipative nonlinear reaction term, and a multiplicative white noise at each node. We proved the existence of a compact global random attractor which pulled back attracts tempered random bounded sets. A challenge problem is to study the long term behavior of the lattice systems with inhomogeneous white noises at each node:

$$
\frac{d u_{i}(t)}{d t}=\nu\left(u_{i-1}-2 u_{i}+u_{i+1}\right)-\lambda u_{i}-f_{i}\left(u_{i}\right)+g_{i}+c_{i} u_{i} \circ \frac{d w_{i}(t)}{d t}, \quad i \in \mathbb{Z} .
$$

Acknowledgements. The main part of this work was done while the first author was visiting Brigham Young University in May 2005. He would like to thank all the people there for the kind hospitality during the stay.

\section{REFERENCES}

[1] V. S. Afraimovich and V. I. Nekorkin, Chaos of traveling waves in a discrete chain of diffusively coupled maps. Int. J. Bifur. Chaos 4 (1994), 631-637.

[2] L. Arnold, Random Dynamical Systems. Springer Verlag, Berlin (1998).

[3] L. Arnold and M. Scheutzow, Perfect cocycles through stochastic differential equations. Probab. Theory Relat. Fields 101 (1995), 65-88.

[4] P. W. Bates and A. Chmaj, On a discrete convolution model for phase transitions. Preprint.

[5] P.W. Bates, H. Lisei, and K. Lu, Attractors for stochastic lattice dynamical systems, Stochastics 85 Dynamics 6 (2006), no. 1, 1-21.

[6] P.W. Bates, K. Lu, and B. Wang, Attractors for lattice dynamical systems. Int. J. Bifur. Chaos 11 (2001), $143-153$.

[7] J. Bell, Some threshhold results for models of myelinated nerves. Mathematical Biosciences 54 (1981), $181-190$.

[8] J. Bell and C. Cosner, Threshold behaviour and propagation for nonlinear differential-difference systems motivated by modeling myelinated axons. Quarterly Appl.Math. 42 (1984), 1-14.

[9] T. Caraballo, P.E. Kloeden and B. Schmalfuß, Exponentially stable stationary solutions for stochastic evolution equations and their perturbation, Applied Mathematics and Optimization 50(2004), 183-207.

[10] T. Caraballo, G. Łukaszewicz and J. Real, Pullback attractors for asymptotically compact nonautonomous dynamical systems, Nonlinear Analysis TMA 64 (2006), no. 3, 484-498.

[11] S.-N. Chow and J. Mallet-Paret, Pattern formulation and spatial chaos in lattice dynamical systems: I. IEEE Trans. Circuits Syst. 42 (1995), 746-751. 
[12] S.-N. Chow, J. Mallet-Paret, and W. Shen, Traveling waves in lattice dynamical systems. J. Diff. Eq. 149 (1998), $248-291$.

[13] S.-N. Chow, J. Mallet-Paret, and E. S. Van Vleck, Pattern formation and spatial chaos in spatially discrete evolution equations. Random Computational Dynamics 4 (1996) 109-178.

[14] S.-N. Chow and W. Shen, Dynamics in a discrete Nagumo equation: Spatial topological chaos. SIAM J. Appl. Math. 55 (1995), 1764-1781.

[15] L. O. Chua and T. Roska, The CNN paradigm. IEEE Trans. Circuits Syst. 40 (1993), 147-156.

[16] L. O. Chua and L. Yang, Cellular neural networks: Theory. IEEE Trans. Circuits Syst. 35 (1988), $1257-1272$.

[17] L. O. Chua and L. Yang, Cellular neural neetworks: Applications. IEEE Trans. Circuits Syst. 35 (1988), 12731290 .

[18] A. Pérez-Muñuzuri, V. Pérez-Muñuzuri, V. Pérez-Villar, and L. O. Chua, Spiral waves on a 2-d array of nonlinear circuits. IEEE Trans. Circuits Syst. 40 (1993), 872-877.

[19] H. Crauel, Random point attractors versus random set attractors. J. London Math. Soc. 63 (2002), $413-427$.

[20] H. Crauel, A. Debussche, and F. Flandoli, Random Attractors. J. Dyn. Diff. Eq. 9 (1997), 307-341.

[21] H. Crauel and F. Flandoli, Attractors for random dynamical systems. Probab. Theory Relat. Fields 100 (1994), $365-393$.

[22] R. Dogaru and L. O. Chua, Edge of chaos and local activity domain of Fitz-Hugh-Nagumo equation. Int. J. Bifurcation and Chaos 8 (1988), 211-257.

[23] G. Da Prato and J. Zabczyk, Stochastic Equations in Infinite Dimensions. Cambridge University Press, 1992.

[24] T. Erneux and G. Nicolis, Propagating waves in discrete bistable reaction diffusion systems. Physica D 67 (1993), $237-244$.

[25] F. Flandoli and H. Lisei, Stationary conjugation of flows for parabolic SPDEs with multiplicative noise and some applications. Stoch. Anal. Appl 22 (2004), 1385-1420.

[26] F. Flandoli and B. Schmalfuß, Random attractors for the 3D stochastic Navier-Stokes equation with multiplicative noise, Stochastics and Stochastic Rep. 59 (1996), 21-45.

[27] P. Imkeller and B. Schmalfuß, The conjugacy of stochastic and random differential equations and the existence of global attractors. J. Dyn. Diff. Eq. 13 (2001), 215-249.

[28] R. Kapval, Discrete models for chemically reacting systems. J. Math. Chem. 6 (1991), 113-163.

[29] I. Karatzas and S.E. Shreve, Brownian Motion and Stochastic Calculus. Springer Verlag, New York (1991).

[30] J. P. Keener, Propagation and its failure in coupled systems of discrete excitable cells. SIAM J. Appl. Math. 47 (1987), 556-572.

[31] J. P. Keener, The effects of discrete gap junction coupling on propagation in myocardium. J. Theor. Biol. 148 (1991), 49-82.

[32] H. Kunita, Stochastic Flows and Stochastic Differential Equations. Cambridge University Press, Cambridge (1997).

[33] J. P. Laplante and T. Erneux, Propagating failure in arrays of coupled bistable chemical reactors. J. Phys. Chem. 96 (1992), 4931-4934.

[34] J. Mallet-Paret, The global structure of traveling waves in spatially discrete dynamical systems. Preprint.

[35] A. Pazy, Semigroups of Linear Operators and Applications to Partial Differential Equations. Springer Verlag, New York (1983).

[36] N. Rashevsky, Mathematical Biophysics. Dover Publications, Inc. Vol 1, New York (1960).

[37] D. Ruelle, Characteristic exponents for a viscous fluid subjected to time dependent forces. Commu. Math. Phys. 93 (1984), 285-300.

[38] M. Scheutzow, Comparison of various concepts of a random attractor: A case study. Arch. Math. 78 (2002), $233-240$.

[39] A. C. Scott, Analysis of a myelinated nerve model. Bull. Math. Biophys. 26 (1964), 247-254.

[40] W. Shen, Lifted lattices, hyperbolic structures, and topological disorders in coupled map lattices. SIAM J. Appl. Math. 56 (1996), 1379-1399.

[41] E. Zeidler, Nonlinear Functional Analysis and its Applications. Vol. I: Fixed-Point Theorems. Springer Verlag, New York (1986).

[42] B. Zinner, Existence of traveling wavefront solutions for the discrete Nagumo equation. J. Diff. Eq. 96 (1992), $1-27$. 
E-mail address, T. Caraballo: caraball@us.es

E-mail address, K. Lu: klu@math.byu.edu or klu@math.msu.edu

(T. Caraballo) Depto. Ecuaciones Diferenciales y Análisis Numérico, Universidad de Sevilla, Apdo. DE Correos 1160, 41080-Sevilla (SPAIN)

(K. Lu) Department of Mathematics, Brigham Young University, Provo, Utah 84602, USA 